\title{
ACTUANDO EN RED. CUERPOS, SESGOS Y GESTIÓN DEL RIESGO EN INTERNET
}

\author{
Nuria Valverde \\ Instituto de Historia, CSIC
}

\begin{abstract}
Science and technology should not be identified merely as risk factors. Both are cultural products and their use contributes to redefine the pressing threats perceived by many civilian groups. Moreover, as strategies of social assembling they have a great impact on the informal networks of knowledge production. This paper analyzes the evolution of depression and electromagnetic fields as scientific and social objects and how are they reconfigured in the web; it aims to explore how the challenges and gains derived from the new electronic tools help to improve problem-solving and risk assessment strategies.
\end{abstract}

KEY WORDS Risk management, internet, depression, electromagnetic fields.
RESUMEN Tecnología y ciencia ya no pueden ser meramente identificadas como factores de riesgo. Ambas son productos culturales y su uso contribuye a redefinir las amenazas que los colectivos perciben como urgentes. Pero hablar del uso de estas estrategias de asamblaje de lo social implica reconocer su impacto en las redes informales de producción de conocimiento. El presente trabajo analiza la evolución de la depresión y los campos electromagnéticos y su presencia en la red con el fin de explorar los retos y aportaciones de las nuevas herramientas de socialización a la resolución de problemas y la gestión de riesgos.

PALABRAS CLAVE Gestión del riesgo, internet, depresión, campos electromagnéticos.

\section{Riesgo y gobernanza}

Hace ya más de 20 años que Mary Douglas y Aaron Wildavsky publicaron su libro, Risk and Culture, sobre la percepción cultural del riesgo tecnológico y medioambiental. La tesis de que no es la percepción del riesgo la que determina la organización social sino que, a la inversa, es el modo de organización el que necesita de formas determinadas de percepción del riesgo, sembraba un -probablemente saludable- escepticismo sobre las aspiraciones de los grupos ecologistas radicales. Pero la gran aportación del libro era la conclusión de que si la selección de riesgos dependia de la elección entre tipos de instituciones sociales, entonces el problema podia replantearse si antes se identificaba qué tipo de cualidades se necesitaban para afrontar riesgos. Las políticas de prevención centralizadas canalizaban una ingente cantidad de recursos hacia un número limitado de objetivos, hipotecando su capacidad de reacción ante los riesgos inesperados. La convicción de los autores era que la diversificación, la variedad y la flexibilidad facilitan la reacción y reconstrucción de los sistemas amenazados. Y fomentar esta capacidad para recuperarse requiere consensos y prácticas de discusión usualmente ausentes de las instituciones caracterizadas por su centralidad, estabilidad e implicación en los procesos de toma de decisiones.
El planteamiento de Douglas y Wildavsky subyace a las actuales reflexiones sobre la evolución de las técnicas de gobernanza. Conscientes de la imposibilidad de prevenir todos los riesgos, las reflexiones se vuelven hacia la capacidad de gestionar lo inesperado. Las estrategias de diálogo cobran peso e importancia. El último informe del proyecto STAGE (Science, Technology and Governance in Europe) subraya la variedad de modelos que se están ensayado actualmente en Europa y advierten que ya no es sostenible una relación didáctica unilateral entre científicos y ciudadanos (vid. Elam \& Bertilsson, 2002). El creciente acceso a una información común permite a los ciudadanos hacer juicios morales sobre la toma de decisiones, mientras reivindican, respaldados por los éxitos de los activistas afectados por el SIDA, que se reconozca que la ciudadanía produce conocimiento relevante. Sin embargo, el camino hacia una democracia deliberativa no parece estarse cimentando a buen paso. Posiblemente, el escepticismo de las instituciones de gobierno hacia la participación ciudadana ${ }^{1}$ está asociado a nuestra incapacidad para dejar de presentar la gobernanza como una estrategia de reparto de poder y ahondar en su eficacia como herramienta para garantizar la capacidad de reacción ante lo imprevisto.

Algunas instituciones comienzan a percatarse de las ventajas de esta nueva orientación. Uno de los efectos de la 
expansión de las soluciones tecnocientíficas modernas es que convierten en excrecencia los conocimientos alternativos. Se produce una reducción de la exteligencia, es decir, del capital cultural colectivo del que cada individuo puede echar mano para solucionar problemas inesperados ${ }^{2}$. Evitar a toda costa esta contracción parece ser la nueva meta de asociaciones como GRAIN, o de los últimos proyectos de FAO, que buscan medidas de rescate de la información tradicional ${ }^{3}$ para combatir las dramáticas consecuencias físicas y sociales en que se sumen las poblaciones cuando quedan desprovistas de estrategias locales viables y eficaces. Pero no sólo se llega a este convencimiento desde el contacto estrecho con las poblaciones del tercer mundo. Las amenazas de la privatización del genoma humano o el creciente número de epidemias asociadas a trastornos psicológicos y del comportamiento nos han sensibilizado hacia la necesidad de preservar el bagaje colectivo y de contemplar los tránsitos entre los cuerpos y las comunidades como un problema de gobernanza. Preservar las pautas y comportamientos locales que contrarrestan un posible riesgo, y sobre todo los mecanismos de diseño de estrategias ante una situación imprevista forma parte de la tarea de articular la erradicación de prácticas injustas, nocivas o dolorosas sin llevarse por delante un conocimiento eficiente que nos beneficia a todos.

Buena parte del conocimiento asociado a la gestión de lo imprevisto emerge en situación de urgencia: media siempre una amenaza de disolución, inconclusión, o fragmentación. En tanto que institución centralizada y jerarquizada, la ciencia ha tendido a minusvalorar los contenidos producidos en estas circunstancias por los colectivos afectados, suponiendo que eran el producto de una magnificación de riesgos menores o de una minimización de riesgos reales, y que, como los productos de la imaginación, caían fuera de la producción científica. Ello ha supuesto el desplazamiento de estos grupos hacia lo que se ha denominado un espacio de abyección: es decir, un espacio social de imposibilidad y sinsentido (Murphy, 2000). Unos colectivos que, sin embargo, necesitan del lenguaje científico y de múltiples herramientas tecnológicas -incluyendo Internet- para definirse como grupo y como individuos. Máquinas y diagnósticos, teorias y modelos, les permiten autoexplorarse y monitorizarse, salir de ese espacio de abyección produciendo una expansión de las prácticas y disciplinas de la objetividad de la que el sistema sanitario, tanto como los afectados, ya no puede prescindir ${ }^{4}$. Todo apunta a que, precisamente en aquellos casos en los que la incertidumbre científica es mayor, es preciso diseñar instrumentos y lenguajes capaces de subsumir la información producida por esos cuerpos dentro de un marco de objetividad sin renunciar a la especificidad: las generalizaciones producidas por los modelos no son más eficientes que las prácticas orientadas a la manipulación concreta de cada uno de ellos (vid. Lettinga \& Mol, 1999).

Los grupos consolidados en torno a la red muestran con su actividad algunos de los caminos posibles para encauzar ese conocimiento. A pesar de las resistencias que ha despertado la absorción de los lenguajes científicos por parte los afectados o los ciudadanos en general, la red ofrece crecientes posibilidades de acceso a publicaciones científicas reconocidas, numerosos recursos de escrutinio, seguimiento y denuncia de intereses, y una vía económica de publicación y discusión a distancia. Todo este instrumental pone a los afectados en disposición, si no de modificar los resultados científicos, al menos, como señala Rodrigue (2001; 2000), de contestar la legitimidad de las técnicas de consenso del riesgo convencionales ${ }^{5}$. Veamos cómo afectan las nuevas herramientas de comunicación electrónica a estos procesos y el impacto que tienen en los modos de concebir la gestión de riesgos.

\section{Razones intimas: nuevas vías de desplazamiento de los problemas de gobernanza}

El desplazamiento de la gobernanza hacia el desarrollo de estrategias de registro e intercambio de prácticas exitosas nos obliga, pues, a volver los ojos hacia esos espacios en los que la transacción de la información se dinamiza y la puesta a disposición de la exteligencia es más evidente: la red.

La red está contribuyendo a la formación de grupos. Cuando Douglas y Windavsky escribieron su libro sólo fueron capaces de ver en la reclutación a distancia de simpatizantes una forma de contribuir al reforzamiento de grupos de naturaleza periférica y sectaria. En principio, la selección de un tema de interés marca la creación de un colectivo caracterizado por la voluntariedad y la tendencia al igualitarismo. Por tanto, según la teoría de estos autores, debería mantener constante la amenaza de un riesgo inminente. Sin embargo, la respuesta no es exactamente esa. Los efectos secundarios juegan un papel central en las listas de correos y los relatos de los afectados por alguna enfermedad (generalmente crónica). Pero no simplemente como materia para 
ofrecer resistencia a un medicamento (pues los efectos secundarios son aceptados mientras no haya alternativa, $y$ la información sobre ellos puede centrarse en el intercambio de consejos y trucos para dulcificarlos). Tienen también una función heuristica: contribuyen a identificar recurrencias y a crear vocabulario para identificar sensaciones, a consensuar posologias y buenas prácticas del paciente, a acumular evidencias en torno a la evolución de la enfermedad 0 al éxito de tratamientos o de estrategias para convivir con la enfermedad ${ }^{6}$. Esta función heuristica tiende a amenazar un mecanismo fundamental para la introducción de nuevos productos y tecnologias: la equivalencia sustancial. Una amenaza que se orienta hacia la exigencia de hacer este concepto más complejo o más apto para mediar entre lo global y lo local.

La equivalencia sustancial establece que si un producto demuestra ser cualitativamente semejante (p.e., por su valor nutritivo, comportamiento bioquímico, capacidad terapéutica, etc.) a otro ya existente, entonces su uso, consumo y regulación puede ser el mismo. El concepto construye una zona de negociación entre objetos "naturales" y artificiales; o dicho de otro modo, entre productos largamente contrastados por el uso y otros fruto de una innovación. Una vez satisfechos los requisitos mínimos de equivalencia, el producto puede pasar al mercado como un sustituto del anterior $^{7}$. Entonces comienzan los problemas. Los consumidores se encuentran con que en muchos casos carecen de recursos para establecer diferencias: los alimentos genéticamente modificados carecen de un olor especifico que los identifique, y es imposible diferenciar una quimera artificial de un mutante natural. Presienten entonces que en esa zona de negociación están ausentes los cuerpos y la especificidad local, y que, por lo tanto, han dejado de ser participes de los procesos de definición del bienestar 8 .

Los ciudadanos se enfrentan pues a la necesidad de reconstruir el cuerpo en espacios de negociación como el que abre la equivalencia sustancial, incorporando en ellos una panoplia más amplia de factores de semejanza y desemejanza. Los referentes locales, las peculiaridades biológicas, los aspectos morales, económicos y culturales entran en acción al definirse la ciudadanía como actor de pleno derecho en la gestión biopolítica (Whatmore, 2002; Levidow \& Murphy, 2002). Una de las estrategias fundamentales de esta reconstrucción es conseguir que las preocupaciones de los consumidores se transformen en incertidumbres científicas, dando lugar a una modificación de los criterios de evidencia. Se le exige asi a la ciencia que produzca estándares más estrictos y, por tanto, menos estables (Levidow \& Murphy, 2002: 17). Para que se produzca esta modificación del modelo científico se necesita información adicional, procedente de puntos muy distantes, que permita detectar posibles síntomas o variaciones en el impacto en las poblaciones humanas.

La ventaja de la equivalencia sustancial es que permite introducir en el mercado productos tecnológicos y científicos con rapidez pero dentro de ciertos márgenes de seguridad. La demanda de incorporación del cuerpo, por su parte, permite rentabilizar los nuevos productos no sólo económicamente (pues evalúa la rentabilidad en función de un espectro más amplio de costes), sino porque la exposición de incertidumbres permite la acumulación de conocimiento local que será útil en el caso de tener que afrontar imprevistos. Pues, ¿qué es lo que incorporan los ciudadanos a estas zonas de negociación? Cuando los ciudadanos identifican conexiones causales arbitrarias, efectos secundarios o carencias en los modelos teóricos, aunque sea de un modo difuso, significa que ya están viviendo en la incertidumbre y que tienen unas estrategias cotidianas para tratar con ella, para afrontar sus limitaciones y para implementar sus ventajas. Rara vez ese conocimiento es individual, aunque, como señalamos, se encuentre geográfica, social o temporalmente disperso. En lo que sigue exploraremos, a través de la historia de la depresión y la de los campos electromagnéticos, en qué forma las redes electrónicas han contribuido a articular ese conocimiento, ofreciendo posibilidades de utilizarlo e incorporarlo a nuestras prácticas de toma de decisiones y gestión del riesgo.

\section{El caso de la depresión}

Se calcula que 121 millones de personas, tanto de paises desarrollados como en vías de desarrollo, padecen esta enfermedad que, junto a la esquizofrenia, es responsable del $60 \%$ de los 8.500 .000 suicidios que anualmente se produ$\mathrm{cen}^{9}$. Combatir la depresión y combatir el suicidio ha llegado en nuestros dias a significar casi lo mismo.

\section{Contextos de emergencia}

Cuando, entre finales de los 50 y principios de los 60, se pusieron en el mercado los primeros antidepresivos (MAOI

ARBOR CLXXXI 715 SEPTIEMBRE-OCTUBRE (2005) 417-433 ISSN: 0210-1963 
y tricicilicos), los especialistas reconocieron que su eficacia no era abrumadora en relación a los placebos. Pero habia buenas razones para adoptarlos. Suponian una alternativa barata a la terapia electroconvulsiva, cuyo coste ascendía a unas 200-500 libras y requería entre 6 y 12 sesiones. Aún así, las recetas se limitaban por temor a los efectos secundarios y prevalecía la convicción de que en un $80 \%$ de los casos la depresión no necesitaba tratamiento químico ${ }^{10}$. Pero a principios de los 60 se produce un cambio. El médico inglés Frank Ayd, involucrado, por encargo de la farmacéutica Merck, en el desarrollo y la comercialización de los tricíclicos, escribe un libro, Recognizing the Depressed Patient (Grune \& Stratton, 1961), destinado a que los no especialistas identifiquen y diagnostiquen a los deprimidos. Merck compró y distribuyó 50.000 ejemplares $^{11}$. Este lanzamiento de la depresión como una enfermedad asociada a un tipo de medicamentos específicos coincidió con otro conjunto de problemas relacionados con la seguridad de los fármacos que, al poner en marcha mecanismos de regulación jurídicos y científicos, determinaron la naturaleza de los procesos de participación ciudadana en los años siguientes.

En efecto, entre 1960 y 1980, periodo en el que se descubren y comercializan los nuevos antidepresivos (los inhibidores selectivos de la recaptación de la serotonina, ISRS), la industria farmacéutica se verá sacudida por varios escándalos a partir de los cuales se regula su producción. Dos de ellos fueron particularmente llamativos: el de la talidomida y el de las benzodiazepinas.

La comercialización precipitada ${ }^{12}$ de la talidomida como tranquilizante en 1957 fue la causa de que en Europa nacieran unos 10.000 bebés con focomelia (atrofia de las extremidades). Circunstancialmente, en los Estados Unidos la Food and Drug Administration (FDA) denegaría en 1961 la autorización para su comercialización, pero la medida fortaleció la confianza en los órganos de control y vigilancia e impulsó que el Congreso aprobase la moción que obligaba a los fabricantes a demostrar la seguridad y eficacia de los medicamentos ${ }^{13}$. También en Europa, a pesar de las diferencias en la gestión y en el impacto sobre el mercado farmacéutico, se crearon comités de regulación similares. En el Reino Unido el desastre dará lugar a la creación del Committee on the Safety of Drugs y, posteriormente, del Committee on the Safety of Medicines. La talidomida habia puesto de manifiesto que las medidas de seguridad en lo que respecta a los medicamentos habian avanzado muy poco desde los años 30, por lo que estos organismos se

ARBOR CLXXXI 715 SEPTIEMBRE-OCTUBRE (2005) 417-433 ISSN: 0210-1963 orientaron hacia el diseño de nuevas fórmulas para garantizar esta seguridad (Ceccoli, 2002: 140).

El Valium, uno de los tranquilizantes menores (del grupo de las benzodiazepinas) más populares de todos los tiempos, entraba en el mercado al mismo tiempo que se desarrolla el escándalo de la talidomida. Entre 1968 y 1987 -y a pesar de las protestas de los grupos antipsiquiátricos- este será el medicamento más recetado de todos los tiempos en el mundo occidental (Tone, 2005: 377-378). A principios de los años 80 , las consecuencias derivadas de la magnitud y el prolongado consumo comienzan a concretarse en demandas por problemas de dependencia, y se pone sobre el tapete la cuestión de la eficacia de las drogas en relación a sus efectos secundarios y la liberalidad en la prescripción. Consumidores y fabricantes emergieron como actores principales en la discusión sobre la responsabilidad en el consumo. Pero mientras los efectos secundarios de la talidomida no admitían gran discusión, establecer los efectos secundarios de las benzodiazepinas requería la transmisión adecuada de los informes sobre cada caso. Tras 17 años de uso, en Gran Bretaña sólo se presentarian 28 informes de reacciones adversas producidas por la abstinencia (Medawar, 1997, § 3.3), a pesar de que en 1988 unas 300 personas en este país reclamaron una compensación por la adicción que habían desarrollado debido al uso prolongado de tranquilizantes ${ }^{14}$. Dada la desproporción entre la información de efectos secundarios transmitida por los médicos y la que, a través de los afectados, llegaba al juez, era evidente que algo en este proceso no estaba funcionando.

Los nuevos antidepresivos heredarán los problemas que subyacen a estos dos escándalos, como la imprevisión sobre el efecto a largo plazo. Precisamente es el riesgo a largo plazo el que de repente equipara en la opinión pública los riesgos medioambientales y los farmacológicos, introduciendo los factores sociales en la discusión sobre éstos (vid. Speaker, 1997: 366ss). En todo caso, ambos habrán contribuido a centrar la atención en tres focos de tensión que emergen en la discusión sobre los riesgos farmacológicos: los modos de elaboración de modelos causales, el grado de indefensión del consumidor y los mecanismos por los que se establece, ajusta y temporiza la posología.

\section{Propiedades, efectos secundarios y riesgo: el papel de los ensayos clínicos}

La fluoxetina, sintetizada por primera vez en 1974, llega al mercado americano como antidepresivo en 1987 (Healy, 
1997: 167-168). Bajo la presión de la mala prensa de los tranquilizantes en los mercados estadounidense y británico, los sindromes de ansiedad pasaron a ser tratados como depresión (Healy, 1997; 2004), y en poco tiempo los ISRS alcanzaron récord de ventas tanto en EEUU como en Euro$\mathrm{pa}^{15}$. Pero este auge no sólo, ni siempre, está asociado a la desconfianza hacia los tranquilizantes.

Desde los años 60, la comprensión de las enfermedades mentales se basa en la extrapolación de los modelos de acción de los psicotrópicos a una patologia (Dupont, 1999: 225; Healy, 1997). La depresión no es una excepción. La serotonina ( $5 \mathrm{HT}$ ) es descubierta como neurotransmisor central en 1955. Sin embargo, no será hasta la década de los 80 cuando se disponga de drogas que permitan manipular eficientemente, y por lo tanto conocer, el sistema del $5 \mathrm{HT}^{16}$ (Healy, 1997: 155). Los primeros pasos para este conocimiento se dan en 1971 cuando se sintetiza el primer inhibidor de la recaptación de la serotonina especifico, la zimelidina. Tras los preceptivos ensayos clínicos, los laboratorios Astra lo lanzan al mercado europeo como Zelmid en $1982^{17}$. En cuanto se verificaron los efectos antidepresivos de la zimelidina se identificó un mercado interesante para los ISRS (Healy, 1997: 168). La fluoxetina salió del limbo de la indecisión sobre sus posibles usos y se definió como un producto específico ajustado a un nuevo modelo de la enfermedad. Aunque esto no significase, paradójicamente, que los demás principios activos, descubiertos al amparo teórico de otro modelo y en otra época, dejasen de ser considerados efectivos.

La incorporación de los ensayos clínicos controlados y ciegos al proceso de control farmacológico es pues importante para explicar cómo el modelo tiene un éxito tan clamoroso en un contexto tan poco propicio y cómo se utiliza en la organización social. Introducidos en la medicina clínica en los 50 para garantizar la eficacia de los nuevos remedios terapéuticos ${ }^{18}$, hasta 1980 las compañias farmacéuticas sólo tendrán que mostrar que el nuevo principio activo funcionaba tan bien como otro ya aprobado ${ }^{19}$. A partir de entonces, se establece que el término de comparación sea regularmente un placebo, además de otro elemento activo. La comparación con el placebo establece la eficacia del medicamento. La comparación con otro principio activo semejante permite valorar si los efectos secundarios (i.e., los riesgos) son desproporcionados en relación a medicamentos ya disponibles. Esto no significa en ningún caso que los resultados de la comparación fueran ni sean evidentes. En
1998 Irving Kirsch y Guy Sapirstein publicaron un análisis de los resultados ofrecidos por el placebo en 19 ensayos clínicos ciegos sobre antidepresivos. El polémico resultado de este estudio fue que el $75 \%$ del efecto de los antidepresivos se debia al efecto placebo, y que los antidepresivos no mostraban un efecto especifico en el tratamiento de la depresión, observándose en otras drogas no antidepresivas una respuesta semejante (Kirsch \& Sapirstein, 1998) ${ }^{20}$.

Al introducirse la dinámica de la comparación para garantizar la eficacia del medicamento, las diferencias mínimas con relación al placebo se contrarrestaban con la demostración de la superioridad (en términos de minimización de efectos secundarios) de un nuevo principio activo sobre otro. Esta estrategia se convirtió en el motor productivo de la industria que, como señala Pignarre, al renunciar a comprender por qué funcionan los medicamentos, renunciaba a la vez a innovar y a que los fármacos fuesen definitivos (Pignarre, 2005: 53). $Y$ en este mismo gesto, mientras las nuevas substancias se hacen más equivalentes, los cuerpos se hicieron intercambiables ${ }^{21}$. Las circunstancias personales, el dolor y el sufrimiento concreto de los pacientes dejaron de ser motivo de atención. Este distanciamiento estaba implicito ya en el diseño de los propios ensayos clínicos que, concebidos para evitar y omitir cualquier sesgo subjetivo por parte de todos los sujetos implicados en el experimento (vid. Kaptchuk, 1998; Hacking, 1988), suprimía el discurso del paciente y la opinión de los expertos sobre la eficacia del medicamento. En definitiva, la aportación de datos por parte del paciente y su interpretación se habia desequilibrado extraordinariamente en relación a unos datos bioquímicos que, sin embargo, resultan ser todavia muy inciertos (Pignarre, 2005: 76).

Además, al delegarse los controles sobre la seguridad de los medicamentos en las industrias farmacéuticas, se potenció indirectamente la comercialización de aquellas sustancias que, con independencia de si respondian o no a una demanda urgente, tenian pocos efectos secundarios $y$, por lo tanto, podian consumirse con tranquilidad. Al mismo tiempo, la justificación del consumo se apoyó en la amplificación de los riesgos. En el caso de los antidepresivos los básicos eran dos: evitar el suicidio y evitar el fracaso social. La gran industria desarrolló herramientas (fundamentalmente basadas en la comunicación y las relaciones públicas ${ }^{22}$ ) que, en última instancia, adoptan la forma de construcción del riesgo de las sectas descrita por Douglas y Wildavsky. 
Y el discurso encontró eco entre la población. La versión simplificada de los mecanismos neuroquímicos según la cual la depresión tenía su causa en los bajos niveles de serotonina y que, por lo tanto, era suficiente corregir dichos niveles para volver a la normalidad convertía la cuestión de la depresión en un mero desequilibrio biológico. Los grupos de afectados se sumaron a esta explicación con comodidad. La principal de las razones, tal como la National Alliance for the Mentally III la enunció, era que esta teoría dejaba claro que "las enfermedades mentales no son el resultado de la debilidad personal, de la falta de carácter o de la falta de refinamiento ${ }^{23}$. Desplazar el estigma asociado a los desórdenes emocionales era una buena razón para abrazarla. El lenguaje científico contribuia a que el paciente pudiese diseñar estrategias para gestionar su enfermedad sin ver involucrada toda su identidad (Dumit, 2003). Y, sin duda, mientras el riesgo de autodestrucción estuviese asociado a desequilibrios neuroquímicos, cerrar filas en torno al consumo de fármacos resultaría en más cohesión social al eximir de responsabilidad tanto a los miembros "desequilibrados" como a las estructuras. Pero en la medida en que la etiqueta de enfermedad mental se extiende a un espectro cada vez más amplio de sintomas y conductas, las personas se ven forzadas a establecer constantes correlaciones entre sus propios cuerpos y las interpretaciones de los sintomas. Esto implica la asimilación de un lenguaje común a legos y expertos, gracias al cual se identifican como factores de conductas peligrosas o indeseables el mismo conjunto de rasgos. Ello facilita el consenso, pero también abre vias a la discrepancia y a la aportación de información que hasta entonces sólo con dificultad era canalizada.

\section{Listas de correo: los efectos de la automonitorización psicológica colectiva}

Hasta los años noventa los antidepresivos no fueron objeto de crítica. Pero al principio de esta década comienzan a oírse las voces de algunos cientificos que siembran la incertidumbre sobre su efectividad y seguridad ${ }^{24}$. También entonces la FDA recibe numerosas quejas sobre los efectos secundarios del Prozac y críticas de toda indole empiezan a aparecer en el seno de todo tipo de asociaciones, sean o no de afectados. Pero es en torno al año 2000 cuando los artículos críticos, como los de Healy, comienzan a ser citados y se consolidan numerosas listas de discusión en torno a las experiencias relacionadas con la suspensión del tratamiento. Entre ellas, The Antidepressant Web, creada en 1998 por
Charles Medawar, que acababa de publicar los resultados de su investigación sobre los efectos secundarios de los ISRS 25 . Seis meses después, The Guardian publica un informe sobre los bajos estándares de los estudios científicos sobre las drogas. Al año la ABC-TV emite dos programas sobre los efectos secundarios de los antidepresivos ${ }^{26}$. Se crean grupos en la red, como ProzacAwareness y Prozac Survivors Support Group, mientras la información sobre depresión y antidepresivos en Internet comienza a adquirir un peso notable ${ }^{27}$. En octubre de 2002 el programa de la BBC Panorama emite un especial sobre los efectos de la paroxetina, titulado "Secrets of Seroxat", que fue visto por cerca de 4,4 millones de personas. Durante su emisión se recibieron 124.000 accesos a la página web del programa, 65.000 llamadas telefónicas y 1.374 correos electrónicos ${ }^{28}$. El análisis de la información aportada por los pacientes y la transmitida a lo largo de 2002 por los profesionales de la medicina señalaba que más pacientes informaban cuando el sistema era directo, $y$ su información era más detallada; mientras que el sistema profesional tendía a omitir información o a clasificarla de tal modo que resultaba difícil crear conexiones ${ }^{29}$. La información global aportada por los pacientes permitía identificar patrones que hasta entonces no habian sido percibidos. Además, revelaban que la distancia respecto a las circunstancias personales había conducido a una discrepancia clara en la percepción de los riesgos (Medawar et al., 2002: 167-168).

Si el contacto por correo electrónico permitia acceder a más información más rápido, las listas de correo demostrarian su eficacia para organizar a las comunidades de afectados. Una de sus ventajas es que permiten mantener la orientación del proyecto sin desviar la atención hacia las ramificaciones de los problemas. ProzacAwareness, por ejemplo, es una lista de correo con 785 suscriptores centrada en los efectos adversos de los antidepresivos y la regulación al respecto ${ }^{30}$, y explícitamente se prohibe publicitar en ella ningún antidepresivo, 0 solicitar información sobre cuál es «mejorn. Sus miembros, ya sean pacientes en tratamiento o familiares, han pasado o están pasando por experiencias negativas relacionadas con el consumo de estos fármacos. Tienen por lo tanto conocimiento de primera mano de los efectos positivos de la medicación, aunque, en general, no consideran que el tratamiento quimico tenga ventajas en relación a los tratamientos alternativos cuando no se prescriben para casos claros de depresión mayor.

Para mantener su sentido, la lista requiere mantener un equilibrio entre lo cada miembro individual aporta y lo que 
la lista ofrece a cambio. Los miembros aportan información continuada y detallada sobre la evolución de sus casos y aventuran teorias o hipótesis sobre las bases fisiológicas de las reacciones que experimentan. Un dato importante es las dosis de los medicamentos que consumen, una información que permite al grupo anticipar algunos efectos y conjeturar el tiempo de disminución de la medicación hasta su retirada total, asi como interpretar de forma coherente las razones de la escalada en la medicación y asociarla a síntomas concretos. Pero comunicar sensaciones para que el grupo evalúe si son sintomáticas requiere determinadas destrezas, porque la comunidad necesita situar la información dentro de unos límites de rigor que le permitan después utilizarla. Por ejemplo, para objetivar la sensación de pérdida de capacidades cognitivas, los pacientes ensayan estrategias (como medir la diferencia de 10 antes y después de la medicación; vid. PA 29200) que permitan a los demás aproximarse al grado de daño que el sujeto experimenta. Pero los datos no se restringen a lo fisiológico. Las relaciones con el médico (el trato, información y atención recibida), el grado de resistencia o apoyo que encuentra en su entorno a la hora de dejar el medicamento, las normas o hábitos sociales que le hacen sentir peor o más desgraciado: todo ello juega un papel importante en la reconstrucción de la enfermedad. Y los miembros de la lista son muy perspicaces a la hora de extraer consecuencias. Por ejemplo, algunos pacientes denuncian que, al ser los antidepresivos tan polivalentes, los médicos los recetan para tratar diagnósticos muy diferentes, y sin embargo no esperan que tengan los mismos efectos secundarios ${ }^{31}$. En esta disociación artificial entre el diagnóstico y las expectativas de efectos secundarios los pacientes encuentran una preocupante inconsistencia con las explicaciones bioquimicas que reciben.

Por su parte, la lista como colectivo enseña a distinguir entre los sintomas de la depresión y los de la suspensión de la medicación (PA 29104); extrae información que permita a los usuarios entender el funcionamiento neuromecánico del cerebro o cotejar sus propias teorias para evitar conexiones falaces (PA 29529); y suministra información sobre los avances políticos del colectivo de afectados. A través de las noticias de nuevos casos y pequeños logros la lista integra a sus individuos en un sistema de evidencias (de ahi la atención que prestan a los asesinatos y masacres presuntamente causadas por las tendencias homicidas y/o suicidas producidas por los ISRS y a los juicios en los que intervienen psiquiatras forenses). Pero también elabora su propio sistema de prácticas y valores que convierte la automonito- rización en un proceso minuciosamente guiado. Desde la lista se definen los protocolos adecuados de disminución de las dosis, las actividades fisicas e intelectuales que se deben evitar o fomentar, estrategias de preparación del entorno familiar ante la "desintoxicación» (PA 29223), se insta a la vigilancia de los hábitos alimenticios, de la gestión del tiempo (para evitar que sea excesivamente estresante), y se comparten las tácticas y experiencias de reintegración social (PA 29222). Por último, el grupo vela por la integridad moral de sus individuos, distribuyendo las responsabilidades entre comportamientos que tienen que ver con la medicación y aquellos que no consideran que estén relacionados. Asi se combaten los sentimientos de culpa por no sentir deseo sexual, o por no haber sido suficientemente fuertes como para superar el síndrome de abstinencia (PA 29715). Pues si las razones para aceptar el tratamiento están relacionadas con evitar el estigma, la renuncia a medicarse o el fracaso a la hora de dejar la medicación generan sus propios abismos morales.

Del mismo modo que los grupos farmacéuticos se perpetúan en la identificación de riesgos, los grupos de afectados aspiran al reconocimiento de la peligrosidad del consumo de los psicofármacos y abogan por una restricción drástica en su uso. Sin embargo, lo que les cohesiona no es un riesgo. Su labor gira en torno a una densa recontextualización (moral, somática y política) de las situaciones de consumo de estos medicamentos. De esta manera contrarrestan la simplificación que permea la publicidad y la prescripción de los mismos. Vigilar y compartir los datos generados por el propio cuerpo y sumarse a un conjunto de prácticas colectivas puede conducir al sectarismo o a la sumisión (Rose, 2003; Dumit, 2003). Pero en el caso de los afectados por antidepresivos, la cuestión de fondo no está anclada en la pureza (espiritual, física o patológica), sino en superación de la abyección a la que se les condena cuando no se les reconoce no ya el derecho a prescindir de la medicación o a tratar la depresión con métodos alternativos, sino la autoridad en el conocimiento de los sintomas y desequilibrios que su propio cuerpo produce. Su forma de reorganizar y hacer habitable la incertidumbre sobre el propio estado de salud, a través de una disección, depuración y jerarquización de causas sociales, bioquímicas y personales es lo que propiamente les identifica. Esta práctica puede contribuir, además, a que las informaciones sobre los efectos adversos de la medicación tengan un resultado coherente. Es decir, a evitar que la publicidad de algún efecto secundario extraordinario tenga un efecto desastroso en el mercado, mientras los informes 
sobre consecuencias generalizadas no consiguen tener impacto. Cuando esto sucede, significa que tanto el sistema de regulación como el publicitario son más sensibles a posibles riesgos abstractos que a la gestión de los problemas concretos y actuales de los pacientes afectados.

\section{El caso de los campos electromagnéticos (CEM)}

La primera vez que se hicieron visibles los efectos biomédicos de los CEM fue en 1979, cuando Nancy Wertheimer y Ed Leeper identificaron una recurrencia: la proximidad de la vivienda habitual de los afectados por leucemia a los tendidos eléctricos de alta tensión. Tras subsiguientes estudios, y a partir de 1989, las autoridades norteamericanas se mantendrán atentas a la posibilidad de los efectos adversos de la exposición a estos campos ${ }^{32}$, y es entonces cuando el discurso precautorio hacia ellos comienza a señalarse como un artefacto mediático. En 1995 el periodista y abogado Michael Fumento, implicado en numerosas disputas sobre la exageración de los riesgos (bio)tecnológicos, publicó un arrebatado artículo contra Paul Brodeur en Reason Maga-

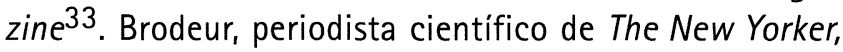
habia publicado en 1989 Currents of Death y en 1993 The Great Power-Line Cover Up, reeditado ese mismo año. El argumento de Fumento se centraba en tres puntos: a) la diferencia entre efectos biológicos y efectos perniciosos para la salud; b) la debilidad de los estudios epidemiológicos; c) los patrones de conexión entre enfermedades. Es decir, que aunque se pudiese demostrar que los campos electromagnéticos tuviesen algún efecto sobre la población, eso no implicaría que tuviera que ser considerado peligroso. Para determinar esta peligrosidad, había que establecer conexiones sólidas entre los efectos y la fuente de riesgo, y los estudios epidemiológicos no estaban en condiciones de hacerlo. Por último, exigía que se ofreciese un modelo explicativo al impacto de los CEM en el organismo humano. Solidez y causalidad son los dos términos en torno a los que girará toda la polémica de los CEM.

\section{Solidez: la emergencia de asociaciones y los límites de la ficción}

Hacia 1993 los Estados Unidos tenían más de 250 asociaciones ciudadanas dedicadas a la cuestión del impacto de los CEM. Aparecen por entonces páginas electrónicas como la Gauss Network (1993); EMF-Link (1994); MicrowaveNews (1995) y EMF-gurú (1996). El número de publicaciones en revistas de impacto se había cuadruplicado con respecto a 1989 (de 17 a 68, según el ISI) y el 63\% de los norteamericanos adultos era consciente de la polémica en torno a los CEM, frente al 31\% de $1989^{34}$. La información estaba en el aire y la expansión de la prevención contra las líneas de alta tensión comenzó a sentirse en el mercado inmobiliario. Algunas sentencias señalaban a las empresas inmobiliarias y a los ayuntamientos, por pasar de puntillas ante la obligación de informar sobre la posible pérdida de valor patrimonial cuando se hallaba próxima una línea de alta tensión, una subestación, o un transformador ${ }^{35}$. El activismo fue particularmente intenso entre $1994-1995^{36}$, y las protestas acabaron por desbordar los espacios jurídicos y políticos, abrumados por la falta de recursos para tratar con problemas marcados por una incertidumbre científica tan alta ${ }^{37}$.

En los EEUU el caso Daubert vs. Merrell Dow Pharmaceuticals (1993) fue la ocasión para elaborar una regulación de la aceptación del testimonio de un experto que por fin escanciara el "hecho científico de la ficción». Como han señalado recientemente Edmond y Mercer (2004), la regulación Daubert ${ }^{38}$-influenciada por los "think tanks» conservadores, como la Atlantic Legal Foundation y el Manhattan Institute, así como por intereses empresarialesevolucionó hacia una ética de la exclusión, que escindía del término ciencia todo conocimiento que no se ajustase a las corrientes científicas sólidamente establecidas sobre las cuales se había consolidado un ideal de verdad. Y si la solidez de un posible riesgo debía adecuarse a un ideal transido por intereses explícitos e implícitos, el juez se convertía en guardián de la interpretación estándar de los procesos sobre la salud pública por encima de la revisión entre pares. Las teorías innovadoras tenían poca cabida, sobre todo cuando era el demandante quien recurria a ellas.

En España, la acumulación de quejas presentadas por distintas asociaciones vecinales ante el Defensor del Pueblo desde 1994, desembocó en 1997 en la presentación al Congreso de los Diputados de un informe y recomendaciones sobre las líneas de alta tensión (LAT) ${ }^{39}$. En dicho informe se afirmaba que uparecia indudablen que los CEM de las LAT y tenían efectos biológicos ${ }^{40}$ y se señalaban como relevantes determinados sintomas (dolor de cabeza, cansancio cróni$c o$, depresión y debilidad muscular). La postura se enfrentaba a las posiciones biofisicas más conservadoras, reivindicaba una interpretación estricta del principio de precaución 
y denunciaba enérgicamente la inadecuación de la normativa de entonces a este principio. Pronto este espacio juridico habria de enfrentarse además a la cuestión de que mientras los estudios que alertaban contra el riesgo de los campos magnéticos se centraban en densidades muy bajas (3-78mG), las medidas precautorias estaban diseñadas para densidades del flujo magnético mucho más altas, por debajo de las cuales no se suponía un daño físico ${ }^{41}$. Sin embargo, en 2001 la Audiencia Provincial de Murcia ratificaría la sentencia del Juzgado de $1^{\text {a }}$ Instancia No 6 contra Iberdrola - la primera en España y una de las primeras de la Comunidad Europea- que la obligaba a indemnizar económicamente a los habitantes de un inmueble y a reducir la penetración de los CEM provocados por un transformador de 1 a $0.3 \mu \mathrm{T}$. La diferencia entre la situación creada por la regulación Daubert y el caso español es que en éste la causalidad es marginal. No se discuten los efectos (ficticios o no) de los CEM sobre la salud, que son considerados como una posibilidad indeterminada. Es el derecho a un entorno no polucionado, no invadido, lo que se reconoce. La solidez, en este caso, se decanta hacia la consolidación de un espacio sano. Pero tanto el recurso al escrutinio de la evidencia científica esgrimida como el desplazamiento del problema de los posibles daños a la invasión de los espacios, tienen una característica en común: los cuerpos siguen ausentes. En la traducción a un discurso sobre la verdad o sobre la propiedad, se desmaterializan.

\section{Causalidad: el cambio de percepción científica y los límites de la casualidad}

A partir de 1999 la inversión en investigación sobre los efectos de los CEM decreció, pero si atendemos al volumen de páginas web, la demanda de información se estaba incrementado ${ }^{42}$. En parte esta expansión podría estar asociada al crecimiento de la red, pero también era consecuencia de un cambio anterior en los métodos científicos. El trabajo de $M$. Feychting y A. Ahlbom de 1992 -popularmente conocido como el informe Karolinska ${ }^{43}$ - y otros posteriores, como el del grupo de Canadá 44 , además de basarse en muestras bastante amplias 45 , introducian nuevos factores como la intensidad del campo magnético - que estaba ausente de los primeros estudios, ceñidos a la proximidad de las líneas de tensión. Con ellos el foco de atención pasa de las líneas de alta tensión al interior de las casas; se desarrollaron métodos para homogeneizar la medida de la exposición (quantitative exposure assessment), se amplia el espectro de la incidencia de la exposición y se intenta determinar el componente bioactivo de los campos ${ }^{46}$. Además, entran en escena nuevos actores, como los electrodomésticos, y nuevos posibles efectos (fatiga, depresión, etc.). El objeto se volvió más penetrante. Y comenzó a prestarse atención al malestar generalizado del que posibles afectados venían quejándose ${ }^{47}$. La idea de integrar la sensación general de salud en los estudios era una forma de objetivar y dar respuesta a una reivindicación dificil de sostener a título individual. Pero sin duda creaba un problema de gestión, y así, cuando algunos trabajos comenzaron a apuntar la posibilidad de vínculos entre los CEM y la depresión, sospechándose una relación con los índices de suicidio, se inició un proceso exhaustivo para evaluar el grado de certeza del conocimiento científico en esta materia. Según el informe 2001 de la International Comission on Non-Ionizing Radiation Protection (Ahlborn, 2001), era preocupante que algunos aspectos básicos en la definición del objeto (la elaboración de un modelo del impacto biológico de los campos electromagnéticos, consenso sobre los métodos y unidades de medida para establecer umbrales de exposición) estuviesen pendientes. Por otro lado, algunos efectos del posible impacto de los CEMs en la salud estaban muy poco desarrollados, de forma que se carecia de base crítica para un análisis comparativo y una evaluación satisfactoria. A pesar de estas carencias, o precisamente por ellas, el estudio de seis años realizados por los epidemiólogos del departamento de salud (DHS) del estado de California (Neutra, Pizzo \& Lee, 2002) se orientó en la dirección de consensuar la percepción del riesgo no de los usuarios, sino de los científicos. El estudio partía de una serie de premisas:

1) Si la polución ambiental no produce efectos detectables en el laboratorio, y se considera un riesgo, no se puede admitir que en relación a los CEM se exijan resultados incuestionables de laboratorio sobre los bioefectos de intensidades menores de $100 \mathrm{mG} .{ }^{48}$

2) Era preciso tener en consideración estudios que, aunque no eran universalmente aceptados, pretendian mostrar los efectos biológicos de intensidades de campos electromagnéticos que desde los modelos producidos por la biofísica resultaban imposibles.

3) En términos generales, los estudios de patologia animal no habian detectado efectos inmunológicos, cancerígenos, o reproductivos.

4) Los estudios epidemiológicos se consideraban exitosos si mostraban que la casualidad era una causa improbable de la asociación entre campos electromagnéticos y la enfermedad considerada. 
La premisa 1 era especialmente importante. Partiendo de ella, el método del DHS tendía a disociar la atribución de riesgo de la disposición de una explicación causal mecanicista o experimental. Esto se lograba evitando que los resultados nulos en los campos de la patología animal o en el enfoque mecanicista afectasen demasiado al grado de certeza. Bastaba reconocer que varias sustancias cancerígenas que afectan al ser humano (humo del cigarro, alcohol, benceno, arsénico) carecieron de un modelo animal durante décadas; y que, del mismo modo, la tesis de que los efectos de los CEM ocurren a nivel químico, celular o pluricelular, pero que no dependen de la presencia de un organismo multicelular intacto, tiene señaladas excepciones. El informe, pues, revisaba toda la literatura relevante sobre el tema aplicando baremos cuantitativos (métodos bayesianos) que desglosaban el grado de creencia de los propios críticos/informantes y su evolución tras la lectura a lo largo de un formulario que recogía sistemáticamente las posibles deficiencias metodológicas y consecuencias disciplinares de las tesis sostenidas en torno a la relación entre los CEM y cada una de las enfermedades o desórdenes que se habian tenido en consideración. El informe, que no unificaba las opiniones de los reviewers participantes, mostrando sus discrepancias, produjo la identificación de los CEM como posible riesgo en más casos que los informes anteriores de otras agencias: incluyendo el cáncer cerebral en adultos, el aborto, o la esclerosis lateral amiotrófica.

\section{Centralidad cultural y multiplicidad: Ia retroalimentación de la convicción}

El informe de California no cerró la controversia en torno a los efectos de los CEMs y las formas de consensuar el riesgo $^{49}$. Tampoco fue el único que reivindicaba la necesidad de reconocer el sesgo impuesto por los modelos en la evaluación de problemas. Ahlbom y Feychting también habian señalado la importancia de someter los resultados cientificos a métodos de evaluación como el de Bayes, que si bien no evitaban el sesgo subjetivo, al menos lo hacían visible. No era sólo una cuestión de objetividad científica, sino de transmisión social de las dinámicas de producción de certidumbre. No parecia razonable que, sin explicación alguna, en investigaciones que presentaban riesgos relativos de 1.7 en la asociación de la aparición de leucemia infantil y la exposición continuada (24h) a intensidades mayores que $0,3 \mu \mathrm{T}(3 \mathrm{mG}) ;$ o de 2.75 en la aparición de esta enfermedad y el uso continuado de mantas eléctricas, se concluyese que

ARBOR CLXXXI 715 SEPTIEMBRE-OCTUBRE (2005) 417-433 ISSN: 0210-1963 el riesgo de padecer la enfermedad no se incrementaba con el aumento de intensidad de los campos magnéticos en las casas de los niños, o que la relación causal entre los campos electromagnéticos de los electrodomésticos y la leucemia era improbable (Ahlbom \& Feychting, 1999). Rescatar las certidumbres del conocimiento tácito, de la inclinación informada, parecia ser la única vía para incorporar los cuerpos afectados.

Los ciberactivistas tuvieron su parte en la consecución de este logro. Al contrario que los afectados por depresión, estos colectivos están orientados a la agitación. Dado que comparten valores y espacios con otros grupos, sus reivindicaciones permean amplios espacios de la red para abundar en el reconocimiento de los riesgos de la electropolución. Su estrategia se centra en el acopio de masivo de información, y en enfatizar a partir de ella las numerosas incongruencias de un sistema que basa la toma de decisiones en la información. Precisamente este es el principio que quieren reforzar páginas como Powerwatch, MicroWaves News, Buergerwelle, la Asociación Sueca de Afectados por Electrosensiblidad (FEB) o EMF Link. Su radicalidad estriba en llevarlo a sus últimas consecuencias: el constante volcado de información, su lectura y revisión, la retroalimentación entre las páginas, contribuyen a azuzar la discusión o, como dicen los autores del libro Black on White, a uarrojar leña al fuegon (Grandlund-Lind \& Lind, 2004). La discusión adquiere proporciones gigantescas, hasta el punto de que nadie puede sentirse ajeno. Bajo el aparente milenarismo de muchas de estas páginas subyace una estrategia de precipitación de la emergencia del objeto.

La consecuencia es que los afectados se ven implicados en una serie de mediciones y análisis del entorno. Si le sale a una escocedura en el lado derecho es preciso averiguar si el vecino del autobús lleva encendido el móvil; probar qué sucede en el propio organismo cuando se desconectan los electrodomésticos; o establecer un programa de medición de radiaciones para verificar si ésta es la razón por la que se encuentra uno peor en una zona u otra de la casa. La reorganización del espacio doméstico puede llegar a ser completa. Todos estos ensayos les permiten, al igual que a los deprimidos, establecer unos rasgos distintivos que les identifican como colectivo. Pero también acumular evidencias para contestar la resistencia oficial a considerar su situación como una patología orgánica y encontrar fórmulas que les permitan llevar una vida agradable. Se anticipan riesgos tal vez demasiado sombrios, pero se presentan cuerpos que 
son reales, como lo muestran las más de 400 cartas de afectados analizados por Grandlund-Lind y Lind. Cuerpos cuya máxima dificultad estriba en que, si no se denuncian las prácticas culturales que imponen límites demasiado estrechos a las conexiones causales que debemos atender en el diseño de nuestras politicas, carecen de posibilidades de integración efectivas fuera de un entorno local muy limitado.

\section{Conclusiones}

La centralidad cultural del discurso científico y la propia peculiaridad del cuerpo bioquímico, reactivo al entorno, emergente, están siendo construidas desde múltiples frentes. La capacidad de los científicos de hacer explícitas las razones y cuantificar la intensidad del cambio de opinión de un experto es importante para garantizar la estabilidad del mismo modelo dentro de un marco multidisciplinar. Como muestra el caso de los CEM, los estudios epidemiológicos dependen de la información de los cuerpos tanto como de la experiencia del propio evaluador: sólo conociendo los mecanismos informales que terminan por sancionar la legitimidad de los grupos de afectados podemos adecuadamente localizar y gestionar los problemas de visibilidad con los que estos se encuentran. Por otra parte, a pesar de tantos esfuerzos por diseñar sistemas de control adecuados sobre los medicamentos, éstos no han dado los resultados deseados. No sólo porque las muertes causadas por los efectos adversos de las medicinas mantengan una tasa muy elevada 50 o porque estemos lejos de resolver el problema del suicidio ${ }^{51}$, sino porque, como muestran en la red los colectivos de afectados por antidepresivos, la automatización de las prácticas terapéuticas distorsiona gravemente el concepto mismo de beneficio.

En ambos casos se señala un punto ciego entre la circulación de información científica, las prácticas de producción de conocimiento individual que genera y los compromisos morales (tanto sociales como científicos) a los que se vinculan y que todavía no sabemos manejar. En cualquier caso, si el conocimiento científico ha dejado de ser un bien de los científicos, ya no podemos prescindir de la topografía de los sesgos. En sus intersticios afloran los espacios donde habita parte de la población y reside nuestra ignorancia. El reto que el espacio electrónico pone en evidencia al permitir la creación de comunidades en torno a sintomas es el de considerar que la ganancia de centralidad cultural del discurso científico debe ir acompañada de soluciones tecnológicas que faciliten su armonización con una realidad compleja. Pues si la realidad se ve afectada por la producción de incertidumbre que desde los cuerpos concretos alimenta el discurso científico, también su construcción cultural desde el ámbito científico requiere que ahondemos en los procesos de quiebra de simetría que le acompañan.

El crecimiento de la exteligencia puede interpretarse como una proliferación de estas rupturas. El panorama que dibuja es un universo menos estable pero más riguroso, menos dócil con los modelos que con las prácticas. Asumir el lado productivo de esta dinámica significa crear los consensos en torno a nuevos instrumentos que permitan seguir los tránsitos de esas rupturas y su coherencia, y así redistribuir tanto la autoridad como el conocimiento. Todavía no sabemos con certeza qué significa tomarse en serio a los grupos de afectados, pero si su número ha dejado de ser limitado y su cohesión ya no se centra tanto en posibles riesgos como en la transacción de prácticas, su proliferación sólo puede contribuir a: 1) esclarecer las relaciones entre cultura y materia, entre registro bioquímico e información subjetiva; 2) orientar el desarrollo tecnológico hacia diseños flexibles que permitan la autonomía de los individuos y una mayor adaptación a lo local. Desde esta perspectiva la dicotomía centro-periferia pierde sentido y la lógica de la toma de decisiones centralizada en función de costes y beneficios se desdibuja. La presencia de estos colectivos en la red contribuye a dotarla de nuevos perfiles al discutir públicamente las prácticas de estimación del riesgo y al crear nuevos espacios de legitimidad en los que negociar a qué escala podemos adoptar medidas que minimicen efectos inesperados o asumir riesgos. 
1 Las recomendaciones 6 y 7 del informe STAGE indican hasta qué punto aún no se ha salvado esta desconfianza (STAGE, 2005).

2 El concepto ha sido acuñado por Stewart \& Cohen, 1999: 243.

3 Véase, p. e., el informe de la FAO, La tradition réinventée en http://www.fao.org/ docrep/007/y5511f/y5511f00.HTM; o el proyecto In Situ, de Perú en http://www. insitu.org.pe/webinsitu/conocimientos.htm

4 Esto es particularmente evidente en el caso de los diabético tipo I (vid. Mol, 2000); pero Dumit (2003) también ha mostrado cómo la monitorización permite a los afectados por depresión disponer de categorías para discriminar una situación de riesgo y controlarla antes de que pierdan su autonomia.

5 Véase, p. e., http://www.emfguru.org/, una de las listas de correo sobre EMFs más conocidas (y que se cerró en 2003) cuyo contenido típico tiene que ver efectivamente con la denuncia de los intereses de los investigadores, o con los sesgos que implica la consideración de una posible fuente de riesgo desde un punto de vista parcial.

6 Akrich y Méadel (2002) han estudiado la emergencia de esta umoral del medicamenton en las listas de discusión electrónicas de enfermos de Parkinson, cáncer y fibromialgia.

7 Un buen ejemplo es el lanzamiento comercial de la soja transgénica Roundup ReadyTM de Monsanto, en el que se reivindicaba -bajo la rúbrica de la "equivalencia sustancialn- la ausencia de diferencias entre los productos no modificados y los modificados genéticamente (Whatmore, 2002:27).

8 Ya Ulrich Beck, en La sociedad del riesgo (1986), habia apuntado la idea de que las intuiciones depositadas en el cuerpo, largamente adiestradas por las pautas culturales locales, fracasan a la hora de abordar los nuevos objetos.
9 WHO, 2001. En Europa un $23,4 \%$ de la población se encuentra afectada por algún tipo de problema de salud mental (EORG, 2003). Los psicotrópicos más utilizados son los ansiolíticos y los antidepresivos, pero se considera que parte de los afectados por desórdenes mentales no reciben tratamiento con psicofármacos y sólo el $21 \%$ de los diagnósticos por depresión estaria medicado (ESEMeD/MHEDEA 2000 investigators, 2004, 109, supl. 420 : 55-64).

10 Medawar (1997: § 2.3) cita los siguientes trabajos: Cole, 1964; Kline, 1964; Leyburn, 1967; Byck, 1975; Baldessarini, 1980.

11 El autor describia los rasgos de una enfermedad hasta entonces difusa y defendia que podia ser diagnosticada por un médico de familia o en un centro de asistencia primaria. Aún así, durante esta década y las dos siguientes los diagnósticos se decantaron la mayor parte de las veces hacia la ansiedad y el stress y el insomnio.

12 Entre la fecha de su sintesis y su puesta en el mercado habian trascurrido apenas tres años (Pignarre, 2005: 49ss).

13 Ello supuso una restricción importante de la entrada de drogas en el mercado americano: si con anterioridad a la aprobación de las nuevas enmiendas la media de nuevas drogas era de 46,2 al año, a partir de entonces descendió a una cifra anual de 15,7 (Timmermans \& Leiter, 2000: 46).

14 Joy Melville (1988) afirmaba que el número de adictos podría ser de 2 millones de personas.
15 Entre 1991 y 1995, el número de recetas por ISRS en Gran Bretaña pasó del 1\% del total de prescripciones (18 millones de libras, un 33\% del gasto en antidepresivos) al 32\% (107 millones de libras, un $73 \%$ del gasto en este tipo de fármacos)(Rose, 2003: 4). En España, donde el consumo de tranquilizantes es de los más altos de Europa, la tendencia ha sido la misma. Entre 1985 y 1994 el consumo de antidepresivos aumentó un 247\% (Alonso et al., 1997). Y entre 1999 y 2003 el consumo de antidepresivos no tricíclicos aumentó un $67 \%$; y los tranquilizantes lo hicieron un 22\%. En 2003 la Seguridad Social prescribió 17.363 .000 envases de depresivos no tricíclicos (grupo N06A3, Grupos terapéuticos y Principios activos de mayor consumo en el Sistema Nacional de Salud durante 2003, Información Terapéutica del Sistema Nacional de Salud, http://www.msc.es/profesional/ biblioteca/recursos_propios/infmedic/docu mentos/vol28_5/vol28_5gruposTerapeuticos03.pdf). Este grupo es más o menos equivalente al que componen la suma de los grupos BNF 4.3.3 y BNF 4.3.4 del British National Formulary. En Inglaterra se dispensaron en 2003 un total de 17.598.400 recetas de estos medicamentos (Prescriptions Cost Analysis: England 2003, UK Department of Health, http:// www.dh.gov.uk/assetRoot/04/08/66/04/ 04086604.pdf). La población española a 1 de 2004 era de 43.197 .684 (INE, http:// www.ine.es/inebase/cgi/axi), y la inglesa de 50.093.800 (UK National Statistics, ONS, http://www.statistics.gov.uk/CCl/ nugget.asp?ID=6).

16 Esto significa que durante los años 70 la enfermedad se explicaba en función de otros neurotransmisores, principalmente las catecolaminas (Healy, 1997: 162-163).

17 Será retirado del mercado, sin entrar en el americano, cuando se informen los primeros casos del sindrome Guillain-Barré (Healy, 1997: 166)

ARBOR CLXXXI 715 SEPTIEMBRE-OCTUBRE (2005) 417-433 ISSN: 0210-1963 
18 Durante la primera época del descubrimiento de los antidepresivos los resultados emanaban de la observación clinica, realizada $\sin$ pautas ni protocolos de control. Véase p.e. el caso de G22355 (Imipramina), en Healy, 1997: 51ss. Sobre el contexto de esta introducción, Healy, 1997: 89, 92; Kaptchuk, 1998: 429-430.

19 Hasta 1980 la FDA no se percató de que este procedimiento no garantizaba la eficiencia, sino un cuadro semejante de efectos secundarios que, sobre todo, respaldaba la comerciabilidad del producto (Healy, 1997:101-102)

20 Análisis posteriores han producido el mismo resultado (véase, p. e., Khan, Warner \& Brown, 2000). Recientemente, y en respuesta a las críticas recibidas por el anterior, Kirsch et al. han emprendido un nuevo análisis centrándose esta vez en los datos enviados a la FDA para conseguir la aprobación de los 6 antidepresivos más prescritos (fluoxetina, paroxetina, sertralina, venlafaxina, nefazodona, citalopram), aprobados entre 1987 y 1999. Los resultados vuelven a ser preocupantes, sólo el $18 \%$ de la acción de la medicación sería efecto de la respuesta al medicamento. (Vid. Kirsch, Moore, Scoboria \& Nicholls, 2002).
21 Esto se refleja en el tipo de corrupción que sufrieron los ensayos clínicos. Las diferencias minimas de eficacia de un medicamento candidato respecto a otro existente exigieron muestras más amplias, con el consiguiente aumento del costo. La reclutación de sujetos se volvió entonces problemática, y no siempre legitima. La investigación realizada por el Senado estadounidense entre 1975 y 1977 reveló que las irregularidades afectaban a la cualificación de los investigadores, a la utilización de datos procedentes de un ensayo clínico diferente, a los sujetos, que en ocasiones eran ficticios, a las dosis administradas, y al suministro de dos 0 más medicamentos en fase de investigación al mismo tiempo. Según Medawar, la situación no era mejor cuando en 1987 se realizaron los ensayos clínicos de la fluoxetina (Prozac): estas prácticas irregulares seguían vigentes $y$, además, los pacientes del ensayo se seleccionaban previamente para excluir a aquellos resistentes al tratamiento y a los que respondían al placebo. Por otro lado, era discutible que los ensayos fueran "ciegosn: los efectos secundarios de muchas drogas eran reconocibles tanto para los investigadores como para los pacientes. (Vid. Pignarre, 2005: 28; Healy, 2004; Healy, 1997: 131; Medawar, 1997, § 2.7 ).

22 Vid. Rose, 2003: 23.

23 Cit. Rose, 2003: 16

24 Apenas tres años después de la entrada en el mercado europeo de Prozac, autores como D. Healy y W. Creaney comienzan a publicar sobre los riesgos de suicidio inducido por el consumo de ISRS (vid. Healy \& Creaney, 1991; Creaney, Murray \& Healy, 1991; Healy, 1991). Según el ISI, el impacto de los articulos de esta época, asi como los de 1994, no se hará notar hasta el año 2000.

25 Los investigadores como Medawar prestaron atención al tema porque "tras 17 años de uso, las benzodiazepinas habian dado lugar a 28 Yellow cards de posibles problemas de abstinencia, mientras que el número de los informes relativos a los SSRI (a Marzo de 1997) estaba en torno a los 1000 y seguian creciendon (Medawar, $1997, \S 3.3)$
26 Emitió dos programas sucesivos, el 24 y 25 mayo de 2000 , sobre la relación de la fluoxetina con la aparición de pensamientos y tentativas suicidas, los problemas de dependencia y abstinencia que presentan los antidepresivos Vid. http://abcnews.go.com/sections/living/Dail yNews/ssri000524.html; http://abcnews. go.com/onair/2020/2020_000825_SSRIwithdrawal_feature.html

27 Es difícil evaluar la evolución de la información en Internet dado de que las herramientas actuales no están desarrolladas por completo. Una búsqueda temática en Internet Archives se circunscribe a un tercio de la base de este archivo. Pero dado que el monto total es ya bastante representativo ( 11 mil millones de páginas) y teniendo la precaución de no tomar las cifras como valores absolutos, sino como identificadores de una tendencia, podemos hacernos una idea de la evolución de la información sobre este tema. Entre enero 1996- mayo 2003 se publicaron 12.739.655 URL relativas a la salud mental. Las páginas relacionadas con la expresión "Clinical depression" ascendieron a 1.411.700, que evolucionaron de la siguiente manera: en 1999, habia 65.100; en 2000, 137.830; en 2001, 596.610. El tema "taking Prozac" ascendió a un total de 649.740, y entre 1999 y 2001 pasó de 31.570 a 196.000 . Por último, el de los SSRIs produjo aproximadamente 148.300 páginas, de las cuales habia 3.808 en 1999, 19.797 en 2000 y 84.487 en 2001.

$28 \mathrm{El}$ análisis de los correos ofreció los siguientes resultados: el 17\% declaraba que los efectos de la medicación eran positivos (de "muy positivo" a "merece la pena tomarlon, 234 correos), el $48 \%$ de "no merece la pena tomarlo" a "altamente limitante" (647), los indecisos ascendian a un 35\% (469) (Medawar, et al. 2002: 161-165).

29 A finales de año, la paroxetina había dado lugar a 8.625 informes oficiales de reacciones adversas en Inglaterra, en total 19.017 reacciones ordenadas en 1067 categorias. De éstas, $1574(8,2 \%)$ se referian a reacciones ante la suspensión de la medicación, dependencia, daños por envenenamiento y conducta suicida (Medawar \& Herxheimer, 2004: 5-19). 
30 En lo que sigue emplearemos las siglas PA para referirnos a esta lista, y citaremos el mensaje con el número que se le atribuye en la lista. Los mensajes han sido traducidos.

31 PA 29106: el paciente informa de que cuando le prescribieron antidepresivos para tratar la migraña y sugirió que éstos podian tener un efecto adverso sobre sus problemas psicológicos, el médico le respondió que no se los prescribía por sus problemas psicológicos, sino por sus migrañas. La respuesta lógica del paciente es: "hacen lo mismo tanto si el tratamiento es para la migraña como para problemas psicológicos".

32 Vid. Nair, Morgan \& Florig, 1989.

33 Publicación periódica de la Reason Foundation, asociación destinada a la promoción y defensa de los valores liberales [http://www.reason.org/faq.html] que recibe en torno a un millón de visitas mensuales [http://reason.com/Reason _Ads.pdf]. El artículo se titula "Shock Journalism: The Junk Reporting Behind the Power Line-Cancer Connection".

34 Según la encuesta realizada en 1993 por la Cambridge Report Research, citada en Sims \& Dent, 2003: 7.

35 En 1993 la Corte Suprema de Nueva York falló a favor de los propietarios que solicitaban una compensación económica por la pérdida de valor en el mercado de sus inmuebles debido a la percepción de los riesgos de los EMF de los tendidos. La sentencia no condicionaba en ningún modo la compensación a que los riesgos estuvieran o no probados, ya que consideró que el que dicho peligro fuese un hecho cientificamente verificable era irrelevante para la cuestión del valor en el mercado. El fallo semejantes se habian producido en Florida, Kansas y California.
36 Vid. Richard W. Woodley, World EMF struggles. Power Lines Struggles Around the World en www.powerlinefacts. com/ Power\%20Line \%20resistence $\% 20$ worldwide.htm. Por ejemplo, la instalación de sendos radares (Terminal Doppler Weather Radar) en dos de los aeropuertos más grandes de US, JFK y La Guardia (Long Island) se paralizó debido a las protestas ciudadanas. Y la construcción por la Nebraska Public Power District de 98 millas de tendido eléctrico de $345 \mathrm{kV}$ entre Pauline y Moore fue llevada a juicio por la asociación POWER (People Organized for Wise Energy Representation), que presentó a Brodeur como experto ante el Natural Resources Committee of the Nebraska State.

37 Desde 1985, se habian registrado más de 100 pleitos por EMF, y asociaciones como ALERT, CAUSE, UCAN, comenzaban a ser reconocidas como interventores y mediadores en la búsqueda de acuerdos y soluciones. En efecto, los fallos favorables a muchos particulares o asociaciones, asi como las decisiones de distintas instituciones, ponian en evidencia el alcance político de la cuestión.

38 La regulación exige que 1 ) la teoría en cuestión haya sido o pueda ser testada; 2) haya sido publicada y sometida a la critica de pares; 3 ) si se trata de una técnica científica, debe expresarse la tasa de error (conocida o potencial) de la misma; 4) debe tenerse en cuenta si dicha teoría 0 técnica está generalmente aceptada en el campo disciplinar en el que se aplique.

39 Recomendación 7/1997, de 4 de febrero, sobre modificación del trayecto de la linea de alta tensión Otero-Ventas-Alcorcón (BOCG. Cortes Generales. VI Legislatura. Serie A. № 213, p. 57). Publicado en la red como Recomendaciones 1997, en www. defensordelpueblo.es/index.asp?destino $=$ informes 3. asp.

40 Idem, 30.
41 Casi todas las medidas de protección recomendadas por las diferentes agencias e instituciones tomaba como umbral máximo de la exposición ocasional $1000 \mu \mathrm{T}(10000 \mathrm{mG})$ y de la exposición continuada $100 \mu \mathrm{T}(1000 \mathrm{mG})$. De acuerdo con la fórmula establecida, a una frecuencia de $60 \mathrm{~Hz}$ la densidad de flujo magnético debía situarse en $83,3 \mu \mathrm{T}$ o 833 mG (vid. ICNIRP, 1998: 30). Estas limitaciones serán recogidas y reafirmadas por la National Radiological Protection Board en su informe de 2004. (Vid. NRPB, 2004) . Pero otras instituciones de gobierno adoptaron por precaución umbrales más bajos, como en el caso de la normativa para nuevas líneas de transmisión eléctrica del gobierno de Florida, que situó los límites máximos entre $150 \mathrm{mG}(15 \mu \mathrm{T})$ y $250 \mathrm{mG}(25 \mu \mathrm{T})$.

42 Según Internet Archive, entre 1996 y 2003 se publicaron unas 374.220 URL relacionadas con la expresión "EMF exposure", 82.530 de las cuales se publicaron entre 1996 y 1999, 26.040 en el año 2000; 92.610 en 2001, con un incremento de aquellas que expresaban un interés en el cáncer de cerebro; y en 2002 se produjeron unas 40.530 .

43 "Magnetic-fields and Cancer in People Residing Near Swedish High-voltage power-lines", Institut Miljomedicin Karolinska Institute, 1992, 2-10; partiendo de estos datos los autores publicarian Feychting \& Ahlbom, 1993.

44 Theriault et al., 1994.

$45 \mathrm{El}$ informe Karolinska se basaba en el estudio de 500.000 individuos (niños y adultos) que hubiesen vivido dentro del radio de 300 metros de un tendido de alta tensión $(220-400 \mathrm{kV})$ en Suecia entre 1960 y 1985 . El trabajo del equipo de Theriault se basaba en 71 casos de leucemia entre 170.000 trabajadores de la Electricité de France-Gas de France activos entre 1978-1989; 45 casos de 31.543 trabajadores de Ontario Hydro activos en 1973-1988; y 24 casos de los 21.749 operarios de la Hydro-Québec entre 19701988. 
46 En 1997 el trabajo de Michaelis et al. sobre 129 casos de leucemia infantil y 328 controles, asi como el de Linet et al., sobre 638 casos y 620 controles parecian definitivamente desplazar la correlación simple del "wire code" a favor de su combinación con mediciones del flujo magnético en el espacio de residencia.

47 Véase, p.e., (Beale et al., 2001), en el que además de hacerse mediciones de los niveles de exposición sobre una muestra de 560 habitantes de Auckland, los investigadores solicitaron a los individuos que respondieran a un test durante una entrevista de 90 minutos. Las conclusiones del estudio fueron que existia una correlación lineal significativa entre el nivel de exposición a campos magnéticos de $50-\mathrm{Hz}$ y la prevalencia de enfermedades crónicas, la sensación de salud fisica general de los afectados, y dos enfermedades: asma y diabetes tipo II.

48 Los autores señalaban, por ejemplo, que los estudios experimentales tienden a producir resultados falsos o negativos; o bien resultados positivos que sugieren relaciones de dosis-respuesta diferentes a aquellos que pueden obtenerse en campos del medioambiente (Neutra, Pizzo \& Lee, 2002: 34).

49 Un año más tarde el Departamento de Protección Ambiental de Florida afirmaba que el informe de California: «es deficiente a la hora de evaluar la falta de datos que lo apoyen desde los estudios de animales y la carencia de un mecanismo biológico plausible que explique cómo la exposición a EMFs puede causar dañon Vid. Florida Department of Environmental Protection, 2003: 3 .

50 Se estima que los efectos adversos por consumo de medicamentos están entre la $4^{\mathrm{a}}$ y $6^{\mathrm{a}}$ causa de muerte en los Estados Unidos. En Noruega, Francia y el Reino Unido suponen, respectivamente, el $11,5 \%$, el $13 \%$ y el $16 \%$ de ingresos hospitalarios (vid. WHO/EDM/OSM, 2002: 7).

\section{BIBLIOGRAFÍA}

51 En los Estados Unidos el indice de consumo de antidepresivos entre 1988 y 1994 fue del 2,5\% (0,7\% ISRS, 1,8\% no-ISRS) (vid. National Center of Health Statistics, 2004: 58-59). Durante este periodo la tasa de suicidios descendió de 12,4 a 12 (por 100.000 hab.). Entre 1999 y 2000 el consumo de antidepresivos fue de $7 \%$ (4,3\% ISRS, 2,7\% no-ISRS), y la tasa de suicidios pasó de 11 a 10,7 (por 100.000 habitantes, datos de 1999 y 2000 , respectivamente (vid. LCWK3, Deaths, percent of total deaths and rates for the 15 leading causes of death in selected groups, by race and sex, en http://www.cdc.gov/nchs/ datawh/statab/unpubd/mortabs/lcwk3_10 .htm) En Francia, que tiene la tasa de suicidio más alta de Europa, el gasto en antidepresivos se multiplicó por 6,7 entre 1980 y 2001, y llegando a suponer en 2001 el 50\% de las ventas de psicotrópicos. (Vid DREES, 2004). Pero la tasa de suicidios (que entre 1950 y 1975 se habia mantenido en torno al 15,5 ) se ha establecido en 1980-1999 en una media del 20 , oscilando entre 22,5 y 17,5 (WHO, Suicide rates by country).
Ahlbom, A. \& Feychting, M. (1999): A Bayesian Approach to Hazard Identification. The Case of Electromagnetic Fields and Cancer, Annals of the New York Academy of Science, 895, 27-33. Versión on-line en www.annalsnyas.org/ cgi/reprint/895/1/27

Ahlbom, A. et al. (2001): Review of the Epidemiologic Literature on EMF and Health, Environmental Health Perspectives, 109 , supplement 6 , 911-933; en http://ehpnet1.niehs.nih. gov/docs/2001/suppl-6/911-933ahlbom

Akrich, M. \& Méadel, C. (2002): Prendre ses médicaments/prendre la parole: les usages des médicaments par les patients dans les listes de discussion électroniques, Sciences Sociales et Santé, 20:1, 89-116

Alonso, M.P., De Abajo, F.J., Martínez, J.J., Montero, D., Martín-Serrano, G. \& Madruga, M. (1997): Evolución del consumo de antidepresivos en España. Impacto de los inhibidores selectivos de la recaptación de serotonina, Medicina Clínica, 108:5; 161-166.

Beale, I.L., Booth, R.J.; Pearce, N.E.; Heriot, S.A. (2001): Association of Health Problems with $50 \mathrm{~Hz}$ Magnetic Field in Human Adults Living Near Power Transmission Lines, Journal of Australian College of Nutritional \& Environmental Medicine, 20:2, 9-12,15,30, reproducida en www.acnem.org/journal/pdf_files/20 2_august_2001/20-2_health _ problems_of_transmission_lines.pdf

Ceccoli, S. (2002): Divergent Paths to Drug Regulation in the United States and the United Kingdom, The Journal of Policy History, 14:2, 135-169

Creaney, W., Murray, I. \& Healy, D. (1991): Antidepressant Induced Suicidal Ideation, Human Psychopharmacology Clinical and Experimental, 6:4, 329-332

Douglas, M. \& Wildavsky, A. (1983): Risk and Culture. An Essay on the Selection of Technological and Environmental Dangers. Berkeley: University of California Press.

DREES (2004): Les ventes d'antidépresseurs entre 1980 et 2001, Études et Résultats, 285, en http://www.sante.gouv.fr/ drees/etude-resultat/er-pdf/er285.pdf 
Dumit, J. (2003): Is It Me or My Brain? Depression and Neuroscientific Facts, Journal of Medical Humanities, 24:1-2, 35-47

Dupont, J.C. (1999): Histoire de la neurotransmission. Paris: Presses Universitaires de France

Edmond, G. \& Mercer, D. (2004): Daubert and the Exclusionary Ethos: The Convergence of Corporate and Judicial Attitudes towards the Admissibility of Expert Evidence in Tort Litigation, Law \& Policy, 26:2, 231-257

ELAM, M. \& BERTILSSON, M. (2002): Consuming, Engaging and Confronting Science: The Emerging Dimensions of Scientific Citizenship, en STAGE (2005), 117-154

EORG-European Opinion Research Group (2003): Eurobarometer 58.2. The Mental Health Status of the European Population (April 2003) http://europa.eu.int/comm/ health/ph_determinants/life_style/ mental_eurobaro.pdf

ESEMeD/MHEDEA 2000 investigators (2004): Psychotropic drug utilization in Europe: results from the European Study of the Epidemiology of Mental Disorders (ESEMeD) Project, Acta Psiychiatrica Scandinavica, 109, (supl. 420), 55-64

Feychting, M. \& Ahlbom, A. (1993): Magnetic-fields and Cancer in People Residing Near Swedish High-voltage power-lines, American Journal of Epidemiology, 138 (7): 467-481

Florida Department of Environmental Protection (2003): Annual Report on EMF Research, en www.dep.state.fl.us/siting/ Programs/electric_magnetic_rpt_2003.pdf

Gillespie, S., Haddad, L. \& Jackson, R. (2001): HIV/AIDS, Food and Nutrition Security: Impacts and Actions, en http://www.ifpri.org/themes/HIV/accscn2 001sp.pdf.

Grandlund-Lind, R. \& Lind, J. (2004): Black on White - Voices and witnesses about Electro-Hypersensitivity - The Swedish Experience, Mimers Brunn Kunskapsförlag, en http://www.feb.se/feb/ blackonwhite-complete-book.pdf Hacking, I. (1988): Telepathy: Origins of Randomization in Experimental Design, Isis, 79:3, 427-451
Healey, D. (1991): What do 5HT Reuptake Inhibitors do in Obsessive-Compulsive Disorders, Human Psychopharmacology Clinical and Experimental, 6:4, 325-328

Healy, D. \&. Creaney, W. (1991): Fluoxetine and Suicide, British Medical Journal

Healy, D. (1997): The Antidepressant Era. Harvard University Press

ICNIRP (1998): Recomendaciones para limitar la exposición a campos eléctricos, magnéticos y electromagnéticos" (hasta $300 \mathrm{GHz}$ ) [traducción de "Guidelines for Limiting Exposure to Time-varying Electric, Magnetic, and Electromagnetic (up to $300 \mathrm{GHz}$ ), Health Physics, vol 74:4, p. 499-522] en www.icnirp.org/ documents/emfgdlesp.pdf

Kaptchuk, T. J. (1998): Intentional Ignorance: A History of Blind Assessment and Placebo Controls in Medicine,

Bulletin of the History of Medicine, 72:3, 389-433

Khan, A.; Warner, H.A. \& Brown, W.A. (2000): Symptom reduction and suicide risk in patients treated with placebo in antidepressant clinical trials: An analysis of the Food and Drug Administration Database, Archives of General Psychiatry, 57: 311-317

Kirsch, I. \& Sapirsetein, G. (1998): Listening to Prozac but Hearing Placebo: $A$ Meta-Analysis of Antidepressant Medication, Prevention \& Treatment, 1 , http://journals.apa.org/prevention/ volume1/pre0010002a.html.

Kirsh, I., Moore, Th., Scoboria, A. \& Nicholls, S.S. (2002): The Emperor's New Drugs: An Analysis of Antidepressant Medication Data Submitted to de U.S. Food and Drug Administration, Prevention and Treatment, 5, article 23, http:// journales.apa.org/prevention/volume5/ pre0050023a.html

Lettinga, A. \& Mol, A. (1999): Clinical Specificity and the Non-generalities of Science. In Innovation Strategies for Neurological Physical Therapy, Theoretical Medicine and Bioethics, 20, p. 517-535
Levidow, L. \& Murphy, J. (2002): The Decline of Substantial Equivalence: how civil society demoted a risky concept. Paper for conference at Institute of Development Studies, 12-13 December 2002, Science and citizenship in a global context: challenges from new technologie, en http://www.polisci. berkeley.edu/faculty/bio/permanent/anse II,c/foodsafety/Levidow\%20Murphy\%20 paper.pdf

Medawar, C., Herxheimer, A., Bell, A. \& Jofre, S. (2002): Paroxetine, Panorama and user reporting of ADRs: Consumer intelligence matters in clinical practica and post-marketing drug surveillance, International Journal of Risk and Safety in Medicine, 15 (3-4), 161-169

Medawar, C. \& Herxheimer, A. (2004): A comparison of adverse drug reaction reports from professionals and users, relating to risk of dependence and suicidal behaviour with paroxetine, International Journal of Risk and Safety in Medicine, 16 (1), 5-19

Medawar. C. (1997): The Antidepressant Web. Marketing depression and making medicines work, International Journal of Risk \& Safety 10:2, 75-126. On line en http://www.socialaudit.org.uk/ download.htm

Melville, J. (1988): Troubles behind the calm - Growing awareness of the scale of tranquilliser addiction, The Guardian, 17 agosto 1988

Mercer, D. (2002): Scientific Method Discourses in the Construction of 'EMF Science': Interest, Resources and Rhetoric in Submissions to a Public Inquiry, Social Studies of Science, 32:2, 205-233

Mol, A. (2000): What Diagnostic Devices Do: the Case of Blood Sugar Measurement. Theorical Medicine and Bioethics, 21: 9-22

Murphy, M. (2000): The 'Elsewhere within Here' and Environmental IIIness; or, How to Build Yourself a Body in a Safe Space, Configurations, 8: 87-120

Nair I., Morgan, M.G. \& Florig, H.K. (1989): Biological effects of power frequency electric and magnetic fields. Technical report. Congress of the United States Office of Technology Assesment. OTABP-E-51. Washington, D.C.: U.S. Government Printing Office, 1989 
National Center of Health Statistics (2004): Chartbook on Trends in the Health of Americans, Health, United States, 2004, en http://www.cdc.gov/nchs/ data/hus/hus04chart.pdf

Nacional Radiological Protection Board [NRPB] (2004): Review of the Scientific Evidence for Limiting Exposure to Electromagnetic Fields $(0-300 \mathrm{GHz})$, Documents of the NRPB, volume 15: 3 , en www.nrpb.org/publications/ documents_of_nrpb/pdfs/doc_15_3.pdf

Neutra, R.R, V. Del Pizzo, V. \& Lee, G.M. (2002): An Evaluation of the Possible Risks From Electric and Magnetic Fields (EMFs) From Power Lines, Internal Wiring, Electrical Occupations and Appliances. California EMF Program. Final Report, en www.dhs.ca.gov/ehib/emf/RiskEvaluation/ riskeval.html

Pignarre, PH. (2005): El gran secreto de la industria farmacéutica. Barcelona: Gedisa.

Rodrigue, C.M. (2000): The Use of the Internet and Web-Based Technology for Space and Geoscience (Mis)Education: New Media in Natural and Technological Hazard Debates, en: http://www. csulb.edu/ rodrigue/aguf00.html.

Rodrigue, C.M. (2001): The Internet in Risk Communication and Hazards Activism, en: wwww.csulb.edu/ rodrigue/ boulder01 talk.html.

Rose, N. (2003): Becoming Neurochemical Selves (Cap. 3 de Biotechnology between Commerce and Civil Society) en www.Ise.ac.uk/collections/sociology/pdf/ Rose-BecomingNeurochemicalSelves.pdf

Sims, S. \& Dent, P. (2003): The Effect of Electricity Distribution Equipment on the UK Residential Property Market, The 2003 Pacific Rim Real Estate Society Conference. Australia, en http:// pandora.nla.gov.au/pan/35642/2003082 1/business.unisa.edu.au/prres/ Proceedings/Proceedings2003/Sims_Den t_The_Effect_of_Electricity_ Distribution_Equipment_on_the_UK_Res idential_Property_Market.pdf

Speaker, S. L. (1999): From "Happines Pills» to "National Nightmare»: Changing Cultural Assessment of Minor Tranquilizers in America, 1955-1980, Journal of the History of Medicine and Other Allied Sciences, 52: 338-376
STAGE (2005): Science, Technology and Governance in Europe. Final Report. February 2005, en www.stageresearch.net/STAGE/documents/STAGE_ Final_Report_final.pdf

Stewart, I. \& Cohen, J. (1997): Figments of Reality. The Evolution of the Curious Mind. Cambridge: Cambridge University Press.

Theriault, G., Goldberg, M., Miller, A., Armstrong, B., Guenel, P., Deadman, J., Imbernon, E., To, T., Chevalier, A., Cyr, D., Wall, C. (1994): Cancer risks associated with occupational exposure to magnetic-fields among electric utility workers in Ontario and Quebec, Canada, and France - 1970-1989, American Journal of Epidemiology 139 (6): 550-572

Timmermans, S. \& Leiter, V. (2000): The Redemption of Thalidomide: Standarizing the Risk of Brith Defects, Social Studies of Science, 30:1, 41-7

Tone, A. (2005): Listening to the Past: History, Psychiatry, and Anxiety, Canadian Journal of Psychiatry, 50:7, 373-380

Vandenbrocuke, J. P. (2002): The history of confounding, Sozial- und

Präventivmedizin, 47, 216-224

Whatmore, S. (2002): Between Production and Consumption: The Monstrous Topicality of 'GM' Food, on line paper, reeditado en Whatmore, S. (2002) Hybrid Geographies. Natures Cultures Spaces. London: Sage, ch. 6

WHO (2001): Fact sheet, $n^{\circ} 265$ : http://www.who.int/mental_health/man agement/depression/definition/en/ WHO/EDM/OSM (2002): Safety of Medicines. A guide to detecting and reporting adverse drug reactions, en http://www.who.int/medicines/library/qs $\mathrm{m} /$ who-edm-qsm-2002-2/esd_safety.pdf 
Relación de números monográficos de próxima publicación:

Ciencia, Tecnología y Valores desde una perspectiva de género. Eulalia Pérez Sedeño (editora).

Investigación científica y tecnológica de la Cultura. Alicia Rodero (editora).

Generación de señas de identidad. Juan Carlos Velasco (editor).

Escritoras españolas del siglo XX. Carmen Simón Palmer (editora).

Filosofía, literatura y psicoanálisis en la Viena fin de siglo. Homenaje a Sigmund Freud. Fernando Bayón (editor).

La revista Arbor está incluida en el apartado de Arte y Humanidades del CITATION INDEX 


\section{NORMAS DE EDICIÓN DE LA REVISTA}

Los autores deben de enviar dos copias de su manuscrito a la sede de la revista: ARBOR Vitrubio, 828006 Madrid ARBOR publica artículos originales, notas y recensiones de libros. Las contribuciones se ajustarán al siguiente formato:

\section{ENCABEZAMIENTO}

Título del artículo, nombre y apellidos del autor, Centro de trabajo, Universidad de procedencia, etc. Dirección postal y dirección de correo electrónico. Resumen del artículo, con una extensión máxima de 150 palabras, en español y en inglés.

Palabras clave del contenido del artículo en español y en inglés.

La extensión del trabajo, salvo excepciones justificadas será de un máximo de veinte páginas tamaño DINA-4, a doble espacio y por una sola cara (Aproximadamente 2.100 caracteres) Todas las páginas deben de estar numeradas.

\section{BIBLIOGRAFÍA}

La bibliografía figurará al final del trabajo por orden alfabético.

Sólo se incluirán las publicaciones que se hayan utilizado y que se citen expresamente en el trabajo. Adoptarán la forma siguiente: Apellidos del autor, nombre del auto lambos en minúsculas), año de publicación entre paréntesis, dos puntos, título del libro en minúscula y cursiva, lugar de edición y editorial.

\section{REFERENCIAS BIBLIOGRÁFICAS}

En el texto del artículo las referencias a otros trabajos se harán indicando el apellido del autor y año de publicación la lo que se añadirá la página exacta si la cita es textual), separados por una coma, entre paréntesis (Gimber, 2003, 20). Si en una misma referencia se incluyen varios autores, se citarán uno a continuación del otro separados por un punto y coma. Si se incluyen varios trabajos del mismo autor publicados en el mismo año, bastará distinguirlos con letras (Gimber, 2003a, 2003b).
Las citas textuales irán entrecomilladas, señalando a continuación entre paréntesis -no a pie de página- el apellido del autor, el año de la publicación y la página correspondiente (Sánchez Vidal, $1999,545-546)$. Si la cita ocupa más de cinco líneas, se presentará en forma de sangrado.

\section{NOTAS A PIE DE PÁGINA}

Las notas y llamadas de texto se numerarán de forma sucesiva y se situarán a pie de página. Para las referencias bibliográficas que hubiera, se seguirán las mismas normas.

\section{TABLAS, GRÁFICOS Y CUADROS}

Las tablas, gráficos o cuadros deberán ir acompañados de su correspondiente título y leyenda y numerados correlativamente.

Los gráficos podrán entregarse dibujados con tinta negra 0 mediante sistemas informático. Las fotografías podrán estar en soporte informático, diapositivas, negativos o copias en papel de buena calidad.

\section{EVALUACIÓN}

Los artículos y notas serán sometidos al criterio de expertos. Una vez aceptados y atendidas las correcciones deberá remitirse su versión definitiva en soporte informático.

\section{SEPARATAS}

Los autores recibirán 25 separatas de cada trabajo. 